\title{
Protein phosphatase PP4 is overexpressed in human breast and lung tumors
}

Bo Wang ${ }^{1, *}$, Ailian Zhao ${ }^{2, ~ *}$, Lingling Sun ${ }^{3, *}$, Xueyan Zhong ${ }^{4,}$, Jianing Zhong ${ }^{1}$, Haibo Wang ${ }^{1}$, Minhua Cai ${ }^{1}$, Jing $\mathrm{Li}^{1}$, Yi Xu${ }^{1}$, Ji Liao ${ }^{1}$, Jianli Sang ${ }^{3}$, Dipanjan Chowdhury ${ }^{5}$, Gerd P Pfeifer ${ }^{4}$, Yun Yen ${ }^{6}$, Xingzhi Xu ${ }^{1}$

${ }^{1}$ Laboratory of Cancer Biology, College of Life Sciences, Capital Normal University, \#105 Xi San Huan Road (N), Beijing 100037, China; ${ }^{2}$ Department of Pathology, Peking University School of Oncology, Beijing 100142, China, ${ }^{3}$ College of Life Science, Beijing Normal University, Beijing 100875, China; ${ }^{4}$ Division of Biology, City of Hope National Medical Center, Duarte, CA 91010, USA; ${ }^{5}$ Department of Radiation Oncology, Dana Farber Cancer Institute, Harvard Medical School, Boston, MA 02115, USA; ${ }^{6}$ Division of Molecular and Clinical Pharmacology, City of Hope National Medical Center, Duarte, CA 91010, USA Cell Research (2008) 18:974-977. doi: 10.1038/cr.2008.274; published online 12 August 2008

\section{Dear Editor,}

Reversible protein phosphorylation by kinases and phosphatases is one of the most common mechanisms in controlling most, if not all, cellular processes [1]. Dephosphorylation of serine/threonine residues is regulated by two distinct groups of functionally diverse serine/threonine protein phosphatases, the PPM family (which includes PP2C) and the PPP family, which includes the type 1 (PP1) and the type 2A (PP2A, PP2B, PP3, PP4, PP5, PP6, and $\mathrm{PP} 7$ ) protein phosphatases. Members of the type $2 \mathrm{~A}$ group of phosphatases (PP2A, PP4, and PP6) are the most closely related in sequence. They are sensitive to inhibition by low doses of okadaic acid (OA) or fostriecin, which distinguishes them from PP1 [1]. The regulation and function of PP2A have been thoroughly investigated. PP2A is composed of a core complex containing the catalytic subunit (C) and the scaffold subunit (A), which interacts with a wide variety of regulatory subunits and interacting proteins that target the AC core dimer to specific substrates and subcellular locations [1]. The assembly of many different types of heterotrimeric holoenzymes accounts for the ability of PP2A, and, possibly, PP4 and PP6, to regulate a wide range of biological processes.

Protein phosphatases 4 catalytic subunit (PP4C) plays an important role in microtubule growth/organization [2], apoptosis [3, 4], tumor necrosis factor signaling [5],

\footnotetext{
*These four authors contributed equally to this work. Correspondence: Xingzhi Xu

Tel: +86-10-68902440; Fax: +86-10-68906307

E-mail: Xingzhi_Xu@mail.cnu.edu.cn
}

and pre-T-cell receptor signaling [4]. The ablation of PP4 leads to embryonic lethality in mice [4]. The human PP4 phosphatase complex, PP4C-PP4R2-PP4R3, was recently identified through mammalian TAP-tagging technique and mass spectrometry [6]. In Saccharomyces cerevisiae, the pph3 (PP4 orthologue), psy2 (PP4R2 orthologue), and yb1046w (PP4R3 orthologue) mutants, but not the pph22 (PP2A orthologue) mutant, are hypersensitive to cisplatin treatment $[6,7]$. Since cisplatin is a replication-dependent DNA-damaging agent [8], this suggests that PP4 is involved in DNA damage signaling and/or repair. Indeed, we recently found that PP4C dephosphorylates $\gamma-\mathrm{H} 2 \mathrm{AX}$ generated during DNA replication [9], while PP2A dephosphorylates $\gamma$-H2AX generated in response to exogenous DNA damaging agents [10].

To further reveal the functions of protein phosphatase PP4, in collaboration with the Bethyl Laboratories Inc, we produced four anti-peptide polyclonal antibodies per target against PP4C and its potential regulatory/binding proteins (PP4R1, PP4R2, PP4R3 $\alpha$, and PP4R3 $\beta$ ). We identified at least one antibody for each target protein, which was suitable for immunoblotting and immunoprecipitation, except that the PP4C antibody was only good for immunoblotting (data not shown). Co-immunoprecipitation assays (Supplementary information, Data S1) showed that PP4C formed three mutually exclusive complexes (PP4C-PP4R1, PP4CPP4R2-PP4R3 $\alpha$, and PP4C-PP4R2-PP4R3 $\beta$ ) in the breast cancer cells MDA-MB-231 (Supplementary information, Figure S1) and MCF7 (data not shown), which were not altered after exposure to DNA-damaging agents such as $\gamma$ radiation, adriamycin, or camptothecin (data not shown). Although PP4R $3 \alpha$ and PP4R3 $\beta$ are close homologues, 
A

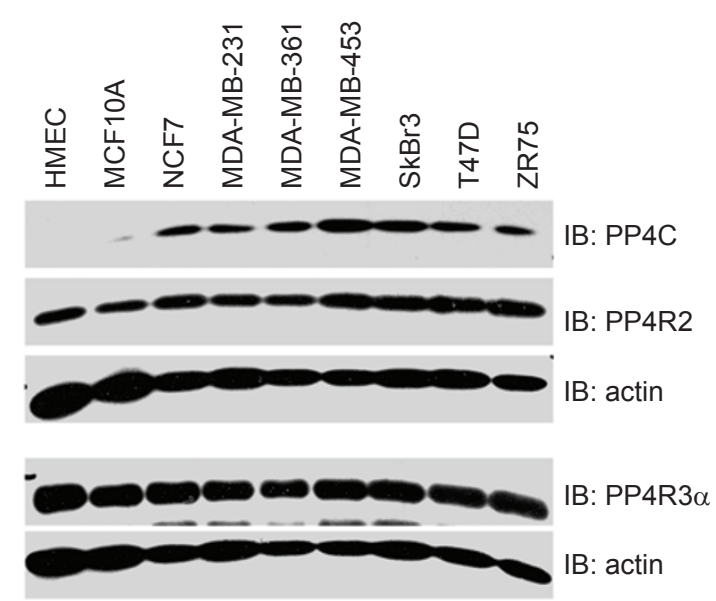

B

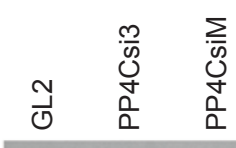

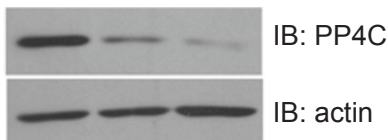

C

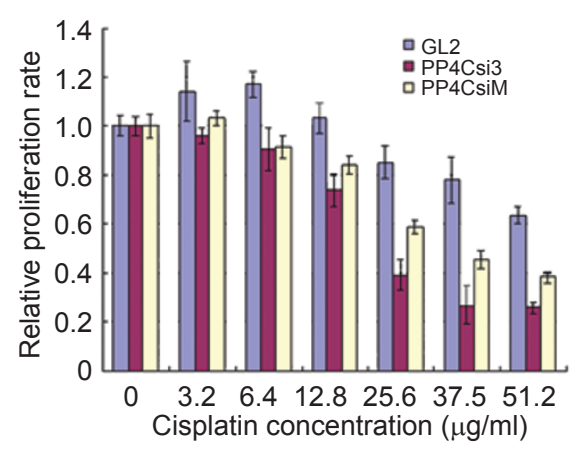

$\mathrm{D}$

PP4C, IDC-III PP4R2, IDC-III PP4R3 $\alpha$, IDC-II

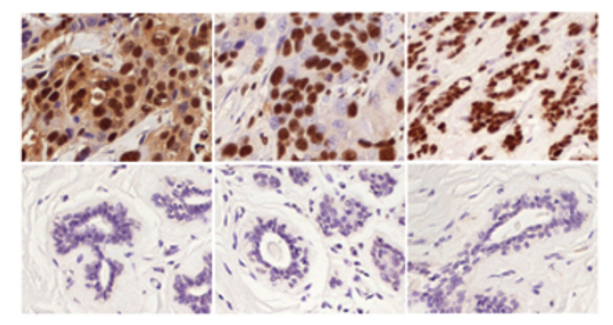

PP4C, benign PP4R2, benign PP4R3 $\alpha$, benign
E

PP4C, WD AD PP4R2, MD AD PP4R3 $\alpha$, PD AD PP4R3 $\alpha$, benign

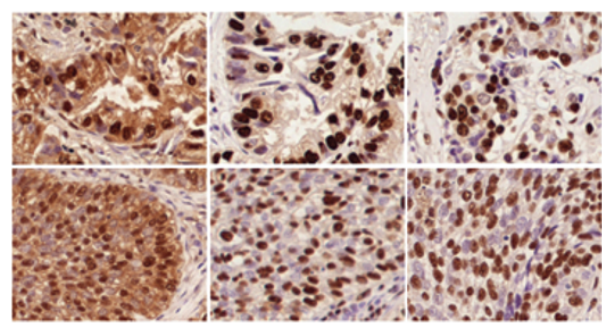

PP4C, MD SQ PP4R2, MD SQ PP4R3 $\alpha, M D$ SQ

$\mathrm{F}$

\begin{tabular}{|c|c|c|c|c|c|c|c|}
\hline & Negative & + & ++ & +++ & Subtotal & \multicolumn{2}{|c|}{ Mann-Whitney U test } \\
\hline PP4C in breast & & & & & & Sum of ranks & Asymp Sig (2-tailed) \\
\hline Carcinomas & 2 & 6 & 8 & 30 & 46 & \begin{tabular}{l|l}
857 &
\end{tabular} & \multirow[t]{2}{*}{0.000} \\
\hline Benign & 23 & 8 & 5 & 1 & 37 & 2629 & \\
\hline PP4R2 in breast & & & & & & & Asymp Sig (2-tailed) \\
\hline Carcinomas & 4 & 13 & 19 & 10 & 46 & 1025.5 & \multirow[t]{2}{*}{0.000} \\
\hline Benign & 29 & 9 & 2 & 0 & 40 & 2715.5 & \\
\hline PP4R3 $\alpha$ in breast & & & & & & & Asymp Sig (2-tailed) \\
\hline Carcinomas & 5 & 15 & 14 & 12 & 46 & 1151 & \multirow[t]{2}{*}{0.000} \\
\hline Benign & 24 & 12 & 3 & 1 & 40 & 2590 & \\
\hline PP4C in lung & & & & & & & Asymp Sig (2-tailed) \\
\hline Carcinomas & 5 & 10 & 17 & 25 & 57 & 1107.5 & \multirow[t]{2}{*}{0.013} \\
\hline Benign & 10 & 4 & 10 & 7 & 31 & 2808.5 & \\
\hline PP4R2 in lung & & & & & & & Asymp Sig (2-tailed) \\
\hline Carcinomas & 1 & 9 & 10 & 37 & 57 & 901.5 & \multirow[t]{2}{*}{0.000} \\
\hline Benign & 5 & 11 & 10 & 5 & 31 & 3014.5 & \\
\hline PP4R3 $\alpha$ in lung & & & & & & & Asymp Sig (2-tailed) \\
\hline Carcinomas & 6 & 8 & 19 & 24 & 57 & 957.5 & \multirow[t]{2}{*}{0.000} \\
\hline Benign & 18 & 2 & 5 & 6 & 31 & 2958.5 & \\
\hline
\end{tabular}


we failed to detect any physical interaction between them (Supplementary information, Figure S1). None of these PP4 subunits interacted with the related phosphatases PP2A and PP6C (data not shown), or PPM phosphatases PPM1D and PPM1G (Supplementary information, Figure S1). We noted that an earlier study [6] identified similar PP4 complexes in epitope-tagged overexpression systems. Our study here demonstrated endogenous PP4 complexes for the first time.

The PP4C gene has been mapped to the middle region of the 16p11.2 locus (Ensembl Gene Report for ENSG00000149923, www.ensembl.org). Gain of DNA copy number within this locus has been reported in invasive breast tumors [11]. Therefore, we sought to determine the expression levels of PP4C and its associated proteins in human breast cancer cell lines by immunoblotting (Figure 1A). Very low levels of PP4C were detected in the human primary mammary epithelial cells (HMEC) and the nontumorigenic epithelial cell line MCF10A. PP4C was readily detectable in the estrogen receptor-positive breast cancer lines MCF7, T47D, MDA361, ZR75, and SkBr3, as well as in the estrogen receptor-negative lines MDA-MB-231 and MDA-MB-453. It was noted that PP4C-associated proteins (PP4R1, PP4R2, PP4R3 $\alpha$, and PP4R3 $\beta$ ) were readily detectable in all the cell lines examined, including HMEC and MCF10A (Figure 1A and data not shown), indicating an additional role for those proteins.

Because deletion of the PP4C orthologue, $P$ PH3, in budding yeast confers hypersensitivity to cisplatin treatment $[6,7]$, we reasoned that inhibition of $\mathrm{PP} 4 \mathrm{C}$ expression might sensitize cancer cells to cisplatin treatment. MCF7 cells were transfected with a control siRNA oligo, a PP4Cspecific siRNA oligo, or a mixture of four PP4C-specific OnTarget plus siRNA oligos (Dharmacon). The expression level of PP4C reduced significantly in both PP4C-specific siRNA oligo transfectants compared to the control siRNA transfectants (Figure 1B). When transfected MCF7 cells were treated with various concentrations of cisplatin, PP4C- depleted cells became more sensitive to cisplatin treatment when its concentration was higher than $12.8 \mu \mathrm{g} / \mathrm{ml}$ (Figure 1C). It should be noted that PP4C depletion reduces cell viability even in undamaged cells (data not shown). Stable knockdown of PP4C using shRNA approach in the lung cancer cells A549 also sensitized them to cisplatin treatment (Supplementary information, Figure S2).

We then sought to determine the expression status of PP4C and its associated proteins in human solid tumor samples. To this end, we tested whether antibodies (normal rabbit IgG and blocking peptides were used as controls) were suitable for immunohistochemistry staining. We found that antibodies for PP4R1 and PP4R3 $\beta$ failed to stain, while antibodies for PP4C (BL3108), PP4R2 (BL3118), and PP4R3 $\alpha$ (BL3121) specifically stained on the formalinfixed and paraffin-embedded tissues (data not shown). Sections of breast ( 46 cancerous lesions and 40 benign lesions) and lung ( 57 cancerous lesions and 31 benign lesions) were stained with these antibodies. A summary of breast and lung sample information is shown in Supplementary information, Table S1. Immunoreactive signals of all three antibodies were evenly distributed in the nucleus, and cytoplasmic staining of PP4C was also observed in both breast (Figure 1D) and lung cancer samples (Figure 1E). Summary of IHC results and statistical analysis are shown in Figure 1F. The nonparametric Mann-Whitney test demonstrated that expression levels of PP4C, PP4R2, and PP4R3 $\alpha$ were significantly higher in cancerous lesions of breast and lung than those in benign lesions of breast and lung (Figure 1F). We further found that 17 out of 40 benign breast samples versus 2 out of 40 breast tumor samples, and 5 out of 31 benign lung samples and none of 57 lung tumor samples were not stained by all three antibodies, whereas 1 out of 40 benign breast samples versus 21 out of 40 breast tumor samples, and 7 out of 31 benign lung samples and 34 of 57 lung tumor samples were strongly stained (IHC scores were ++ or +++ ) by all three antibodies (Supplementary information, Table S2). We did not examine any significant

Figure $1 \mathrm{PP} 4 \mathrm{C}$ is overexpressed in human breast and lung tumors. (A) PP4C is overexpressed in human breast cancer cell lines. Total cell lysates were extracted from breast cell lines and immunoblotted for PP4C, PP4R2, PP4R3 $\alpha$, and $\beta$-actin. (B, C) Inhibition of PP4C expression sensitizes cancer cells to cisplatin treatment. MCF7 breast cancer cells were transfected twice with a control siRNA oligo (GL2) or PP4C-specific siRNA oligo (PP4Csi3 or PP4CsiM) using oligofectamine. Transfectants were trypsinized $24 \mathrm{~h}$ after the second transfection and seeded into a 96-well plate along with different final concentrations of cisplatin as indicated. Each concentration point was repeated at least 4 times. Expression of PP4C is shown in panel B, and relative proliferation rate after cisplatin treatment is shown in panel C. (D) Representative images of expression patterns of PP4C-PP4R2-PP4R3 $\alpha$ in breast tumors. Immunohistochemical staining with PP4C, PP4R2, or PP4R3 $\alpha$ antibodies in breast cancer cells (upper panel) and mammary epithelial cells in breast benign lesions (bottom panel) is shown. IDC: invasive ductal carcinoma. (E) Representative images of expression patterns of PP4C-PP4R2-PP4R3 $\alpha$ in lung tumors. Immunohistochemical staining with PP4C, PP4R2, or PP4R3 $\alpha$ antibodies in lung adenocarcinoma (first three images in the upper panel) and lung squamous cell carcinoma (bottom panel) is shown. Immunohistochemical staining with PP4R3 $\alpha$ in a lung benign lesion is shown in the 4th image in the upper panel. AD: adenocarcinoma; SQ: squamous cell carcinoma; WD: well differentiated; MD: moderately differentiated; PD: poorly differentiated. (F) Summary of IHC results and statistical analysis. 
correlation between staining intensity and differentiation status of either breast samples or lung samples due to the limited case number in each pathological category. Our finding indicates a potential link between the PP4 expression levels and tumorigenesis.

We noted the discrepancy that overexpression of PP4R2 and PP4R $3 \alpha$ appeared in human primary breast tumors but not in the breast cancer cell lines. A simple explanation is that cancer cell lines are grown on plastics for many passages and do not reflect primary tumors. It is also possible that the observed differences are due to different epitope exposure in the formalin-fixed and paraffin-embedded samples.

Platinum-based anticancer drugs such as cisplatin, carboplatin and oxaliplatin have been used successfully to treat many solid tumors including breast and lung cancer [8]. However, development of cellular resistance dramatically compromises treatment success [8]. Resistance may be attributed to several factors, including enhanced DNA repair, tolerance to platinum-induced DNA damage, and alterations in signal transduction pathways [8]. We recently demonstrated that PP4C is the phosphatase that dephosphorylates $\gamma-\mathrm{H} 2 \mathrm{AX}$ generated during DNA replication [9]. High levels of PP4C in cells may dephosphorylate replication block-associated $\gamma-\mathrm{H} 2 \mathrm{AX}$, promote DNA replication regardless of replication block, escape cisplatin-induced apoptosis, and accumulate DNA mutations, which further predispose cells to genomic instability. Indeed, we showed here that PP4C-PP4R2-PP4R3 $\alpha$ were over-expressed in human primary breast and lung tumors and inhibition of PP4C expression sensitized breast and lung cancer cells to cisplatin treatment.

Aberrant protein phosphorylation has been associated with many human diseases, including tumors [1]. Protein kinases have been extensively studied and currently are the second largest drug target for the pharmaceutical industry. Many inhibitors of protein kinases have been successfully applied in cancer treatment. It is noted that aberrant phosphorylation may result from aberrant dephosphorylation. Therefore, protein phosphatases are being recognized as promising therapeutic targets, and our results suggest that PP4C may be one of such promising targets.

\section{Acknowledgments}

We thank Eric W McIntush from the Bethyl Laboratories for providing the PP4 antibodies for testing. We thank the other members of Xu's laboratory for their help. This work was supported by the startup fund from Capital Normal
University, National Natural Science Foundation of China (30570371, 90608014 and 30711120570), Program for New Century Excellent Talents in University (NCET-06-0187), Beijing Natural Science Foundation Program and Scientific Research Key Program of Beijing Municipal Commission of Education (KZ200810028014), and Funding Project for Academic Human Resources Development in the Institutions of Higher Learning under the Jurisdiction of Beijing Municipality (PHR(IHLB)) to XX, and a grant from National Institutes of Health, USA (R01CA88873) to GPP.

\section{References}

1 Moorhead GB, Trinkle-Mulcahy L, Ulke-Lemee A. Emerging roles of nuclear protein phosphatases. Nat Rev Mol Cell Biol 2007; 8:234-244.

2 Toyo-oka K, Mori D, Yano Y, et al. Protein phosphatase 4 catalytic subunit regulates Cdk1 activity and microtubule organization via NDEL1 dephosphorylation. J Cell Biol 2008; 180:1133-1147.

3 Mourtada-Maarabouni M, Kirkham L, Jenkins B, et al. Functional expression cloning reveals proapoptotic role for protein phosphatase 4. Cell Death Differ 2003; 10:1016-1024.

4 Shui JW, Hu MC, Tan TH. Conditional knockout mice reveal an essential role of protein phosphatase 4 in thymocyte development and pre-T-cell receptor signaling. Mol Cell Biol 2007; 27:7991.

5 Mihindukulasuriya KA, Zhou G, Qin J, Tan TH. Protein phosphatase 4 interacts with and down-regulates insulin receptor substrate 4 following tumor necrosis factor-alpha stimulation. $J$ Biol Chem 2004; 279:46588-46594.

6 Gingras AC, Caballero M, Zarske M, et al. A novel, evolutionarily conserved protein phosphatase complex involved in cisplatin sensitivity. Mol Cell Proteomics 2005; 4:1725-1740.

7 Wu HI, Brown JA, Dorie MJ, Lazzeroni L, Brown JM. Genomewide identification of genes conferring resistance to the anticancer agents cisplatin, oxaliplatin, and mitomycin C. Cancer Res 2004; 64:3940-3948

8 Rabik CA, Dolan ME. Molecular mechanisms of resistance and toxicity associated with platinating agents. Cancer Treat Rev 2007; 33:9-23.

9 Chowdhury D, Xu X, Zhong X, et al. A PP4-phosphatase complex dephosphorylates gamma-H2AX generated during DNA replication. Mol Cell 2008; 31:33-46.

10 Chowdhury D, Keogh MC, Ishii H, Peterson CL, Buratowski $\mathrm{S}$, Lieberman J. gamma-H2AX dephosphorylation by protein phosphatase 2A facilitates DNA double-strand break repair. Mol Cell 2005; 20:801-809.

11 Stange DE, Radlwimmer B, Schubert F, et al. High-resolution genomic profiling reveals association of chromosomal aberrations on $1 \mathrm{q}$ and $16 \mathrm{p}$ with histologic and genetic subgroups of invasive breast cancer. Clin Cancer Res 2006; 12:345-352.

(Supplementary Information is linked to the online version of the paper on the Cell Research website.) 